\title{
Non-Contrast Renal MR Angiography: Value of Subtraction of Tagging and Non-Tagging Technique
}

\author{
Takehiro Shimada, MD, Makoto Amanuma, MD, Ayako Takahashi, MD, and Yoshito Tsushima, MD
}

\begin{abstract}
Purpose: The aim of this study was to examine the usefulness of the subtraction technique of non-contrast renal magnetic resonance angiography (MRA) between tagged and non-tagged data collection.

Material and Methods: We performed renal MRA on eleven healthy volunteers using a 3T MRI unit. For renal MRA, a three dimensional balanced type steady-state free precession (SSFP) sequence (True FISP, Siemens) was used with diaphragmatic navigator gating. We tried to acquire selective arterial images by subtracting black-blood images (tagged images, on which arterial longitudinal magnetization was nearly zero by selective inversion of upper-stream aortic flow) from bright-blood images (non-tagged images, on which arterial flow is bright due to inflow effect). For analysis, two radiologists independently evaluated the visual quality of the axial and coronal targeted maximum intensity projection images (MIP) of original bright-blood MRA and subtraction MRA.

Results: Visualization of the main stem of the renal arteries and their 1st branches were satisfactory on both techniques, and there was no statistically significant difference. The score of 2 nd branch appeared superior with the subtraction method, but only the right side showed a statistically significant difference $(P<0.01)$. Visualization of small intraparenchymal arteries was significantly superior with subtraction method on both sides.

Conclusion: We tried to improve selective demonstration of renal arterial branches using subtraction technique. Although full sequence optimization was not performed, this pilot study showed this technique to be slightly time-consuming but superior in visualization of peripheral branches and possibly more sensitive in detecting small vessel abnormalities.
\end{abstract}

Keywords: magnetic resonance angiography (MRA), renal artery, non-contrast technique, 3T

MRI, SSFP imaging

\section{INTRODUCTION}

I

$n$ the diagnosis of renal artery stenosis, catheter angiography has been considered the gold standard, but

Department of Diagnostic, Interventional Radiology and Nuclear Medicine, Gunma University School of Medicine, Maebashi, Gunma, Japan

Received: September 22, 2011; Accepted: January 29, 2012 Corresponding author: Takehiro Shimada, MD. Department of Diagnostic, Interventional Radiology and Nuclear Medicine, Gunma University School of Medicine, 3-39-15 Showa-machi, Maebashi, Gunma 371-8511, Japan

Tel: +81-27-220-8612, Fax: +81-27-220-8612

E-mail: cgt@minos.ocn.ne.jp contrast-enhanced computed tomographic angiography (CTA) and magnetic resonance angiography (MRA) have replaced it in recent years. ${ }^{1-3)}$ Catheter angiography is a highly invasive technique, and iodinated contrast materials carry the risk of side effects such as renal toxicity and allergic reactions. ${ }^{4}$ Gadolinium contrast agents also carry the risk of acute side effects, and NSF (nephrogenic systemic fibrosis) is also a concern in patients with renal dysfunction. $^{5,6)}$

More recently, MRA without contrast agents has become increasingly common in the clinical setting, not only in head and neck region, but also in the body and extremities. ${ }^{7,8)}$ Excellent image quality has been reported in renal MR angiography on 1.5T MR units, and the 
Table 1 Imaging parameters for steady-state free perception

\begin{tabular}{lc} 
TR/TE/FA & $1.4 / 1.68 / 90$ \\
TI & $1.28 \mathrm{sec}$ \\
receiver bandwidth & $783 \mathrm{~Hz} / \mathrm{pixel}$ \\
slab thickness & $100 \mathrm{~mm}$ \\
slice thickness & $0.9 \mathrm{~mm}$ \\
field of view & $251 \times 350 \mathrm{~mm}$ \\
resolution & $0.9 \times 1.15 \times 1.27$ \\
parallel imaging factor & 2 \\
\hline
\end{tabular}

usefulness of MRA of the head in higher field units has been established. ${ }^{9)}$ Lanzman et al. reported the usefulness of non-contrast renal MRA performed on a 3 Tesla unit. ${ }^{10,11)}$ The report suggests superior image quality in higher field units is due to both an increase of blood signal and a decrease of retroperitoneal tissue signal. Blood flow signal from the inferior vena cava and renal vein are suppressed by inversion pulses, but inadequate suppression may occur in some cases due to higher velocity blood flow. These may increase signal intensity around the renal arteries, impairing depiction of peripheral branches on non-contrast MRA.

In contrast-enhanced MRA, subtraction technique between 3D slabs before and after contrast administration is routinely performed for improving vascular visualization. ${ }^{12,13)}$ In this technique, if data acquisition timing is not optimal and renal parenchyma becomes enhanced, selective visualization of the renal artery is impeded. However, with appropriate data acquisition timing, excellent selective visualization is possible. On the other hand, the subtraction technique is also feasible on non-contrast MRA, by obtaining images with and without tagging of upper-stream arterial flow. ${ }^{14)}$ Subtraction MRA may, in fact, be more suitable for this technique than contrastenhanced MRA, because there is no major difference in the renal parenchyma signal between images with and without flow tagging for the former. The aim of this study was to examine the usefulness of subtraction technique of non-contrast renal MRA.

\section{Material and Methods}

This study was approved by our institutional review board, and informed consent was obtained from all subjects.

We performed renal MRA on eleven healthy volunteers (seven men and four women; age range, 24-49 years; mean age 30.6 years; body weight range, 45 to $80 \mathrm{~kg}$; mean, $60.3 \mathrm{~kg}$ ) using a 3T MRI unit (Magnetom Trio 3T,
Siemens AG, Erlangen, Germany) in Gunma University Hospital from March 2011 to May 2011. An eight-channel phased array coil was used with a spinal matrix coil. For renal MRA, a three dimensional steady-state free precession (SSFP) sequence (True FISP, Siemens) was used with diaphragmatic navigator gating. For navigator gating, a two-dimensional selective pencil-beam excitation pulse was used to detect the position of the right hemidiaphragm during normal breathing. Data were obtained during expiration, during which diaphragmatic motion would be minimal. Navigation pulses were placed to avoid excitation of the right kidney.

Detailed imaging parameters are shown on Table 1. For all MRA studies, a single three-dimensional slab was placed in a transaxial plane. We tried to acquire selective arterial images by subtracting black-blood tagged images from bright-blood non-tagged images. The bright-blood images are the same as those of the commonly used non-contrast MRA technique, depicting the arterial flow entering the imaging slab as high signal. Background signal suppression was performed by a selective inversion pulse covering the slab. After the 180-degree pulse, the background static tissue recovers and its longitudinal magnetization decreases toward the null point. To suppress the fat tissue signal, an additional frequency selective fat saturation pulse was applied just before data acquisition. The black-blood images were acquired in an identical manner, except for additional suppression of arterial inflow by applying a tagging pulse covering the heart.

The resulting time interval between the first inversion pulse and effective echo time was set at 1280 msec. $^{15,16)}$ Finally, we subtracted the two image sets, and visualization of renal arterial branches was subjectively analyzed.

For analysis, two radiologists independently evaluated the visual quality of the axial and coronal MIP image set. Original bright-blood MRA and subtraction MRA were mixed and randomly ordered for evaluation. The image quality was evaluated at four segments: a) main trunk of renal artery; b) 1st branches (ventral and dorsal branch); c) 2nd branches; d) arcuate arteries. The quality of visualization was scored using a three-point-scale: 3 , continuity and luminal dimension of artery can be evaluated with confidence; 2, artery is depicted, but difficult to evaluate continuity and luminal dimension with confidence; 1, poor (arteries are not visible or difficult to evaluate). Statistical comparison was made with Wilcoxon rank test. A P-value less than 0.05 was considered statistically significant. 
Table 2 Statistical evaluation of visualization score

\begin{tabular}{|c|c|c|c|c|c|c|c|c|}
\hline Part of renal artery & $\underset{\text { trunk }}{\text { Right main }}$ & $\begin{array}{l}\text { Left main } \\
\text { trunk }\end{array}$ & $\begin{array}{c}\text { Right 1st } \\
\text { branch }\end{array}$ & $\begin{array}{l}\text { Left 1st } \\
\text { branch }\end{array}$ & $\begin{array}{l}\text { Right 2nd } \\
\text { branch }\end{array}$ & $\begin{array}{l}\text { Left 2nd } \\
\text { branch }\end{array}$ & $\begin{array}{l}\text { Right arcu- } \\
\text { ate artery }\end{array}$ & $\begin{array}{l}\text { Left arcu- } \\
\text { ate artery }\end{array}$ \\
\hline bright-blood mean score & 2.73 & 3.00 & 2.36 & 2.73 & 1.82 & 2.00 & 1.18 & 1.18 \\
\hline subtraction mean score & 3.00 & 2.91 & 2.64 & 2.82 & 2.36 & 2.36 & 1.91 & 1.64 \\
\hline$P$ value & NA & NA & NA & NA & $<0.01$ & NA & $<0.01$ & $<0.01$ \\
\hline
\end{tabular}
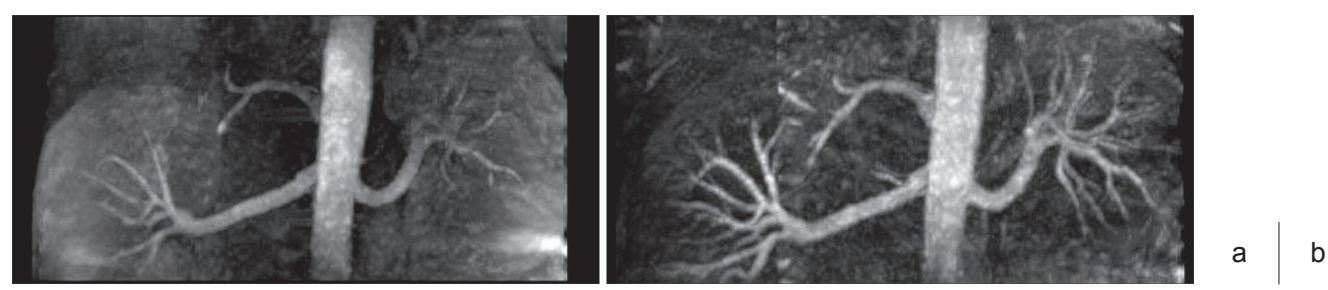

Fig. 130 year-old male volunteer. Coronal maximum intensity projection (MIP) images of original bright-blood MRA (a) and subtraction MRA (b). Though main trunk and 1st branches of renal arteries in both images are depicted equally, 2nd and more peripheral branches are more clearly demonstrated on subtraction MRA.

\section{Results}

Visualization of the main stem of the renal arteries and their 1st branches were satisfactory on both techniques, and there was no statistically significant difference. The score of 2 nd branch appeared superior with the subtraction method, but only the right side showed a statistically significant difference $(P<0.01)$. Table 2 shows the results of statistical evaluation of visualization score. Visualization of small intraparenchymal arteries was significantly superior with subtraction on both sides (Fig. 1). High intensity of the inferior vena cava was seen in six out of eleven cases on bright-blood images, overlapping and sometimes obscuring the 1st or 2 nd branches of the right renal artery on coronal images. On the other hand, there was no observable signal from the inferior vena cava on subtraction images (Fig. 2). Actual imaging time of bright-blood images and black-blood images were 6:29 to 9:35 (mean time 7:59) and 5:27 to 11:02 (mean time 7:28).

\section{Discussion}

The renal artery is a major branch of the aorta, and various vascular lesions may develop in this vessel. Renovascular hypertension is most frequent cause of secondary hypertension, and diagnosis of the disease is very important clinically. ${ }^{17)}$ Atherosclerotic renal artery stenosis tends to induce a narrowing of the main stem lumen, ${ }^{18)}$ while fibromuscular dysplasia usually involves the distal part of main stem. ${ }^{19)}$ Polyarteritis nodosa occurs in the peripheral branches of the renal artery. ${ }^{20)}$ Thus, it is important to visualize the entire renal artery, including the small intraparenchymal branches.

Three-dimensional fast gradient echo with contrast media has been the mainstream of renal MRA. However, since nephrogenic systemic sclerosis (NSF) associated with the use of gadolinium contrast agents has been widely reported, it is virtually impossible to use contrast agents in patients with renal impairment. ${ }^{5,6)}$ While renovascular hypertension caused by renal artery stenosis does not necessarily lead to renal failure, atherosclerosis is the most common disease of the systemic arteries, making it a frequent cause of renal impairment.

Non-contrast MRA appears attractive as a solution of these problems. Recent developments of non-contrast MRA techniques are remarkable. In addition to the conventional time-of-flight and phase contrast techniques, use of the ECG-gated partial-Fourier fast spin echo technique for evaluating the upper and lower extremity arteries has become clinically widespread. ${ }^{7,8)}$ In renal MRA, the steady-state data collection described above, combined with respiratory gating, can provide high-resolution blood vessel images without respiratory movement. ${ }^{16,21-25)}$ We tried to completely suppress the 

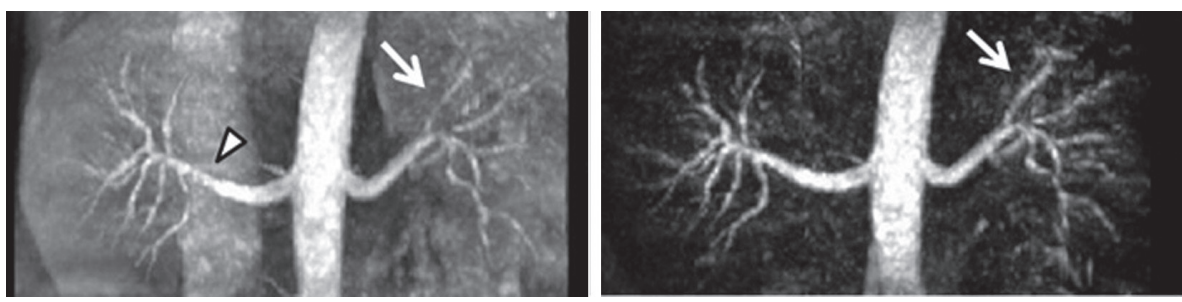

Fig. 229 year-old female volunteer.

Coronal MIP image in the original image (a) demonstrates a focally obscured right renal arterial main stem due to overlapping IVC (arrowheads). On the other hand, the continuity of both renal arteries is preserved at subtraction image (b).

The continuity of $1^{\text {st }}$ and other peripheral branch of left renal artery is better depicted in subtraction image compared with bright-blood image (white arrows).

background signal of both images before subtraction. If the imaging slabs were completely identical, the signal suppression of venous structure or renal parenchyma before subtraction would not be so important. However, to avoid possible misregistration or intestinal peristalsis between images, we thought it would be best if the background signal were reduced as much as possible, even before the subtraction.

In this study, although the proximal renal arterial segments were readily demonstrated on the conventional technique, smaller peripheral branches were more confidently evaluated on subtraction images. The results were considered to be due to superior background suppression and resulting higher contrast on subtraction MRI.

The major drawback of the subtraction method is prolongation of imaging time. Simultaneous imaging of black-blood images and bright-blood images by interleaved mode would be desirable but unrealistic due to the nature of the steady-state technique, and two different acquisitions are needed. Gross patient motion between the two sets of images would cause spatial misregistration, resulting in artifacts possibly interfering with image assessment. However, the signal of renal parenchyma is not necessarily high, and the signal of surrounding adipose tissue is well suppressed, making misregistration effect considerable enough to prevent depiction of vessels less likely to occur. The problem of imaging time may eventually be overcome by more advanced hardware or more sophisticated data acquisition techniques.

There are several limitations in this study. All subjects were healthy and relatively young. Non-contrast MRA depends on the flow velocity of target vessels, but renal artery flow tends to be slower in patients (including but not limited to those with renal impairment). Furthermore, the success rate of navigator gated MR images tends to lower in patients and the elderly. Therefore, misregistration artifact due to respiratory gating would likely be higher in the actual clinical setting.

Second, the timing of inversion pulse application was not optimized on this study. Although we used the same imaging parameters both for bright blood and black blood images, optimal parameters would not necessarily be identical. The bright blood technique requires increased signal of distal branches, so a longer TI time may be suitable. On the other hand, the black blood technique requires the perfect nulling of arterial signal, so a shorter TI might be preferable. Detailed optimization would be necessary for actual clinical use, and we are currently investigating sequence optimization.

\section{Conclusion}

We tried to improve selective depiction of renal MRA with subtraction. Although it is slightly time-consuming and its clinical utility may be currently limited, it provides superior visualization of peripheral branches even in this pilot study. Further sophistication of the technique would make it even more sensitive in detecting small vessel abnormalities and promising for clinical use.

\section{REFERENCES}

1) Slanina M, Zizka J, Klzo L, et al. Contrast-enhanced MR angiography utilizing parallel acquisition techniques in renal artery stenosis detection. Eur J Radiol 2010; 75: e46-50. [Medline] [CrossRef]

2) Nchimi A, Brisbois D, Materne R, et al. Free-breathing accelerated gadolinium-enhanced MR Angiography in the Diagnosis of Renovascular Disease. AJR Am J Roentgenol 2009; 192: 1531-7. [Medline] [CrossRef]

3) Soulez G, Pasowicz M, Benea G, et al. Renal artery 
stenosis evaluation: diagnostic performance of gadobenate dimeglumine-enhanced MR angiographycomparison with DSA. Radiology 2008; 247: 273-85. [Medline] [CrossRef]

4) Shiragami K, Fujii Z, Sakumura T, et al. Effect of a contrast agent on long-term renal function and the efficacy of prophylactic hemodiafiltration. Circ J 2008; 72: 427-33. [Medline] [CrossRef]

5) Cowper SE, Robin HS, Steinberg SM, et al. Scleromyxoedema-like cutaneous diseases in renaldialysis patients. Lancet 2000; 356: 1000-1. [Medline] [CrossRef]

6) Broome DR. Nephrogenic systemic fibrosis associated with gadolinium based contrast agents: a summary of the medical literature reporting. Eur J Radiol 20081; 66: 230-4. [Medline] [CrossRef]

7) Fan Z, Sheehan J, Bi X, et al. 3D noncontrast MR angiography of the distal lower extremities using flow-sensitive dephasing (FSD)-prepared balanced SSFP. Magn Reson Med 2009; 62: 1523-32. [Medline] [CrossRef]

8) Cukur T, Lee JH, Bangerter NK, et al. Non-contrastenhanced flow-independent peripheral MR angiography with balanced SSFP. Magn Reson Med 2009; 61: 1533-9. [Medline] [CrossRef]

9) Fushimi Y, Miki Y, Kikuta K, et al. Comparison of 3.0- and 1.5-T three-dimensional time-of-flight MR angiography in moyamoya disease: preliminary experience. Radiology 2006; 239: 232-7. [Medline] [CrossRef]

10) Lanzman RS, Kröpil P, Schmitt P, et al. Nonenhanced free-breathing ECG-gated steady-state free precession 3D MR angiography of the renal arteries: comparison between 1.5 T and 3 T. AJR Am J Roentgenol 2010; 194: 794-8. [Medline] [CrossRef]

11) Shimada T, Amanuma $M$, Takahashi A, et al. Navigator-gated non-contrast renal MR angiography: qualitative and quantitative comparison of $3 \mathrm{~T}$ and $1.5 \mathrm{~T}$ images. Kitakanto Med J 2011; 61: 483-7. [CrossRef]

12) Watanabe Y, Dohke M, Okumura A, et al. Dynamic subtraction contrast-enhanced MR angiography: technique, clinical applications, and pitfalls. Radiographics 2000; 20: 135-52. [Medline]

13) Leung DA, Pelkonen P, Hany TF, et al. Value of image subtraction in 3D gadolinium-enhanced MR angiography of the renal arteries. J Magn Reson Imaging 1998; 8: 598-602. [Medline] [CrossRef]

14) Miyazaki M, Isoda H. Non-contrast-enhanced MR angiography of the abdomen. Eur J Radiol 2011; 80: 9-23. [Medline] [CrossRef]
15) Glockner JF, Takahashi N, Kawashima A, et al. Noncontrast renal artery MRA using an inflow inversion recovery steady state free precession technique (Inhance): comparison with 3D contrast-enhanced MRA. J Magn Reson Imaging 2010; 31: 1411-8. [Medline] [CrossRef]

16) Utsunomiya D, Miyazaki M, Nomitsu Y, et al. Clinical role of non-contrast magnetic resonance angiography for evaluation of renal artery stenosis. Circ J 2008; 72: 1627-30. [Medline] [CrossRef]

17) Rimmer JM, Gennari FJ. Atherosclerotic renovascular disease and progressive renal failure. Ann Intern Med 1993; 118: 712-9. [Medline]

18) Kawarada O, Yokoi Y, Morioka N, et al. Renal artery stenosis in cardio-and cerebrovascular disease: renal duplex ultrasonography as an initial screening examination. Circ J 2007; 71: 1942-7. [Medline] [CrossRef]

19) Das CJ, Neyaz Z, Thapa P, et al. Fibromuscular dysplasia of the renal arteries: a radiological review. Int Urol Nephrol 2007; 39: 233-8. [Medline] [CrossRef]

20) Ozaki K, Miyayama S, Ushiogi $Y$, et al. Renal involvement of polyarteritis nodosa: CT and MR findings. Abdom Imaging 2009; 34: 265-70. [Medline] [CrossRef]

21) Shimada $K$, Isoda $H$, Okada $T$, et al. Non-contrast-enhanced hepatic MR angiography with true steady-state free-precession and time spatial labeling inversion pulse: optimization of the technique and preliminary results. Eur J Radiol 2009; 70: 111-7. [Medline] [CrossRef]

22) Krishnam MS, Tomasian A, Deshpande V, et al. Noncontrast 3D steady-state free-precession magnetic resonance angiography of the whole chest using nonselective radiofrequency excitation over a large field of view: comparison with single-phase 3D contrastenhanced magnetic resonance angiography. Invest Radiol 2008; 43: 411-20. [Medline] [CrossRef]

23) Maki JH, Wilson GJ, Eubank WB, et al. Steady-state free precession MRA of the renal arteries: breath-hold and navigator-gated techniques vs. CE-MRA. JMRI 2007; 26: 966-73. [Medline] [CrossRef]

24) Katoh M, Buecker A, Stuber M, et al. Free-breathing renal MR angiography with steady-state freeprecession (SSFP) and slab-selective spin inversion: initial results. Kidney Int 2004; 66: 1272-8. [Medline] [CrossRef]

25) Mohrs OK, Petersen SE, Schulze T, et al. High-resolution 3D unenhanced ECG-gated respiratory-navigated MR angiography of the renal arteries: comparison with contrast-enhanced MR angiography. AJR Am J Roentgenol 2010; 195: 1423-8. [Medline] [CrossRef] 